\title{
Morphology, electronic and optical properties of Si nanowires
}

\author{
Pil-Sook Grace Kim,* G. A. Botton,* and T. K. Sham** \\ * Brockhouse Institute for Materials Research, McMaster University, Hamilton, ON, L8S 4L8 \\ Canada \\ ** Department of Chemistry, University of Western Ontario, London, ON, N6A 5B7 Canada
}

In recent years, considerable efforts have been made in the fabrication and characterization of silicon nanowires (SiNWs) due to their unique properties which show high potential for applications in nanoelectronics. SiNWs can be prepared by various techniques [1-3]. The size and morphology of the nanowires, as well as their electronic and optical properties, vary depending on the growth conditions. However, all SiNWs are inevitably capped with a layer of silicon oxide. SiNWs exhibit weak and complex luminescence with emission wavelengths ranging from 420 to $820 \mathrm{~nm}$ due to the wide variation in morphologies [4]. In general, the variations in the luminescence have been ascribed to, or are combination of, the quantum confinement effect, the defects surrounding the crystalline core, the silicon oxide surface, and the silicon-silicon oxide interface [4]. In this report, we present morphology, electronic and optical properties of SiNWs using SEM, TEM, and synchrotron based X-ray absorption spectroscopy and X-ray excited optical luminescence (XEOL) techniques. Synchrotron based XEOL technique is element and excitation channel specific which has proven useful in studies of optical properties of various light emitting materials [5].

Thermal evaporation of $\mathrm{SiO}$ was used to synthesize SiNWs on an n-type $\mathrm{Si}(100)$ wafer and on porous silicon (prepared by electrochemical etching). The SiNWs were synthesized in a double furnace setup with a quartz tube reactor using source temperature of 800 and substrate of $1000^{\circ} \mathrm{C}$.

SEM images, shown in FIG. 1, reveal the formation of nanowires, which exhibit substrate dependent morphology. The SiNWs condensed on the quartz tube are short $(\sim \mu \mathrm{m})$ and thin, $\sim 50 \mathrm{~nm}$ diameter, compared to the thicker nanowires $(>100 \mathrm{~nm}$ diameter) on $\mathrm{Si}(100)$ (denoted $\mathrm{SiNW} / \mathrm{Si}$ ). The nanowires which condensed on the porous silicon (SiNW/PSi), were the thinnest ( $<50 \mathrm{~nm}$ diameter). FIG. 2(a) shows the XEOL recorded for the three different SiNW specimens with excitation photon energy above the oxygen K-edge $(550 \mathrm{eV})$. The XEOL from SiNW and SiNW/PSi are very similar to other silicon nanowires previously reported, such as oxide-capped silicon nanowires or an alternate Si nanoparticle-silicon oxide chain [4]. In all three spectra, two emission bands can be observed at $460 \mathrm{~nm}(2.7 \mathrm{eV})$ and a broad peak in the range of 500-600 $\mathrm{nm}$. The luminescence at 2.7 $\mathrm{eV}$ can be attributed to the neutral oxygen vacancy in the oxide. Luminescence of SiNW on Si wafer sample excited across the oxygen K-edge is shown in FIG. 2(b). The emission profile remains the same regardless of the excitation energies. However, the intensity of luminescence at $460 \mathrm{~nm}$ increases significantly from below the edge $(530 \mathrm{eV})$ to above the edge $(550 \mathrm{eV})$, whereas the intensity of luminescence at $550 \mathrm{~nm}$ does not show significant change. From the oxygen K-edge results we can at least exclude the association of the Si oxide to the origin of the weak emission at $550 \mathrm{~nm}$. Another interesting observation is that only the SiNW/Si specimen exhibits an additional feature at $\sim 300 \mathrm{~nm}$, which is characteristic of emission from defects of silicon oxide glass. The corresponding oxygen K-edge XANES in TEY, FLY and PLY (photoluminescence) in zero order, as well as partial PLY, at selected wavelength for all three samples will be further discussed to understand the origin of the luminescence from these samples [6]. 


\section{References}

[1] Y.F. Zhang et al., Appl. Phys. Lett. 72 (1988) 1835.

[2] A. Morales et al., Science. 279 (1998) 208.

[3] J.D. Holmes et al., Science. 287 (2000) 1471.

[4] T.K. Sham et al., Phys. Rev. B 70 (2004) 045313.

[5] P.S. Kim et al., J. Am. Chem. Soc. 123 (2001) 8870.

[6] This research was supported by the NSERC. The aid of Dr. Yongfeng Hu of CSRF is gratefully acknowledged.
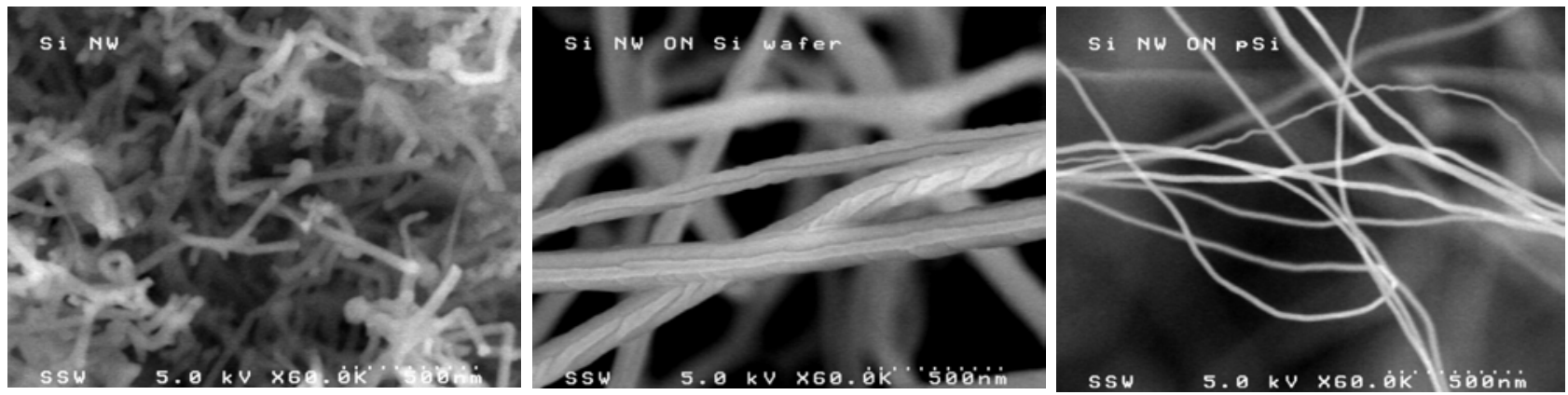

FIG. 1. SEM images of the products, from left to right: free standing SiNW condensed on the quartz tube, SiNWs collected on a $\mathrm{Si}(100)$ wafer and a porous silicon substrate.
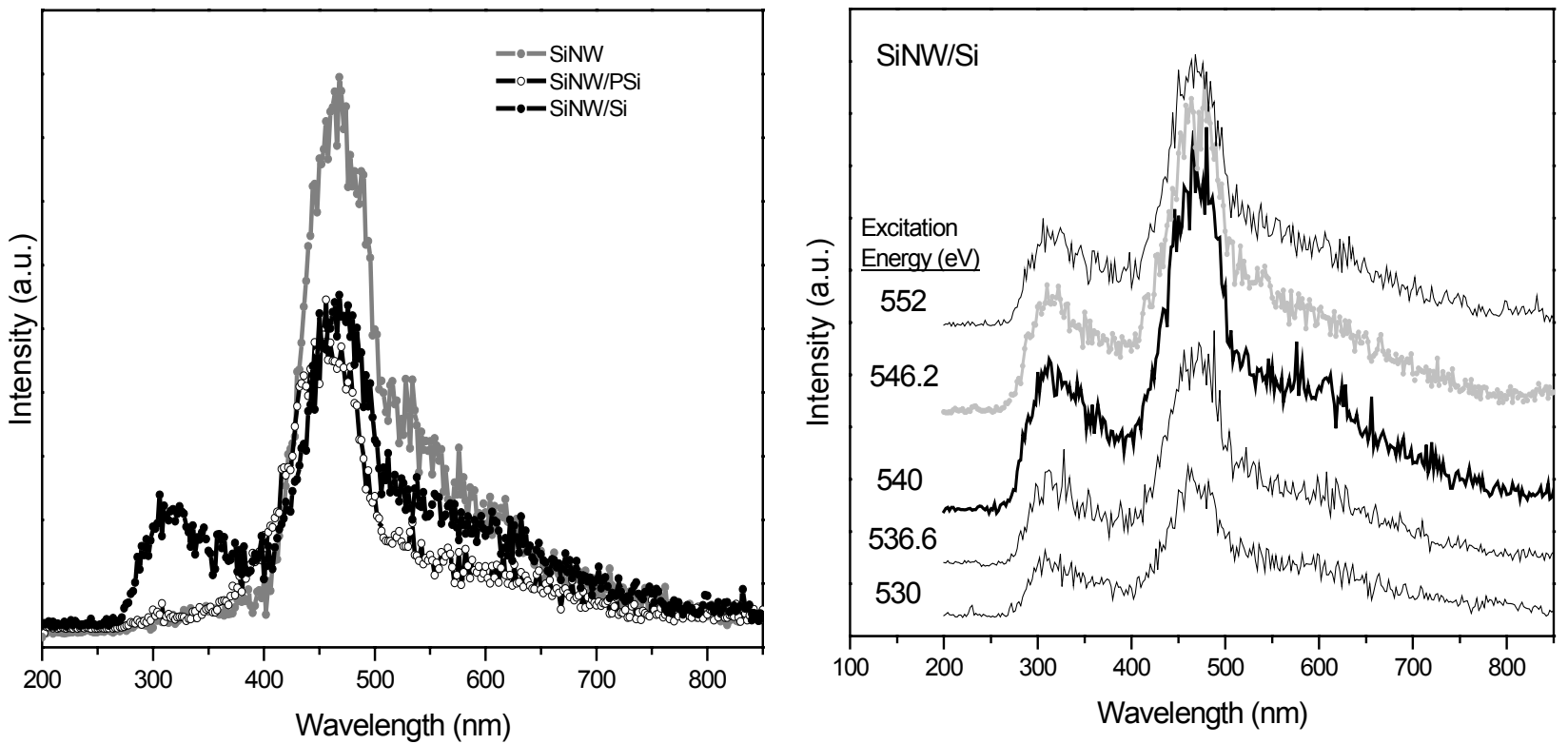

FIG. 2. (a) XEOL spectra of the three Si nanowire samples, SiNW, SiNW/PSi, and SiNW/Si, excited at $550 \mathrm{eV}$. (b) XEOL spectra of SiNW on Si wafer at the selected excitation energies across O K-edge. 